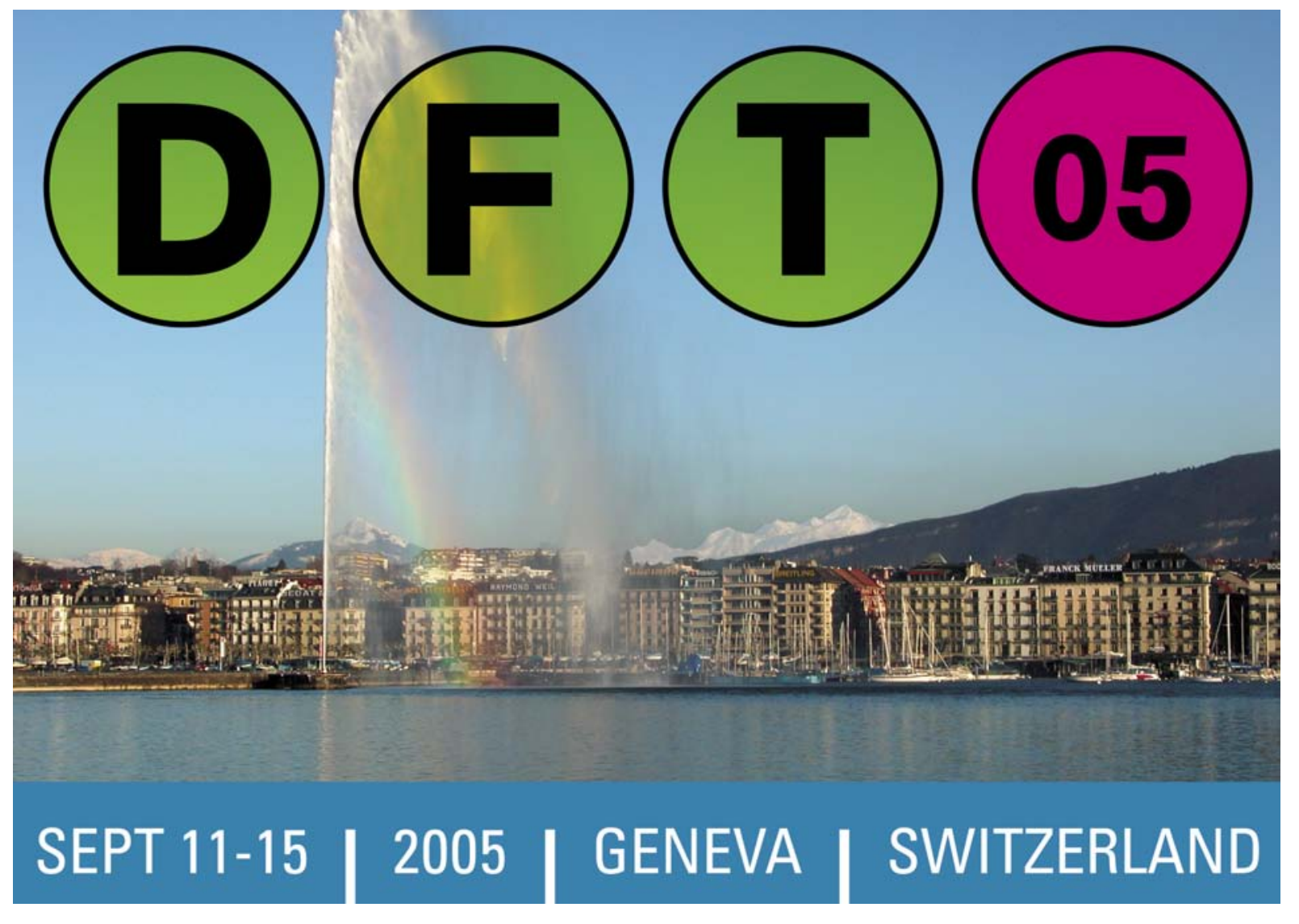

\title{
ABSTRACTS OF ORAL PRESENTATIONS
}

\section{Legend}

11-I16: Invited Lectures

C1-C14: Oral Contributions 
Invited lectures

\title{
"Nearsightedness of Electronic Matter" \\ E. Prodan* and W. Kohn*
}

*University of California, Santa Barbara, California 93106 University of Southern California, Los Angeles, California 90089

\begin{abstract}
"Nearsightedness of Electronic Matter" (NEM) is a general principle which can be viewed as underlying concepts such as the chemical bond and transferability. Very qualitatively speaking, it describes the fact that: the magnitude of the effect a perturbing potential $\mathrm{w}\left(\mathrm{r}^{\prime}\right)$, no matter how strong and extended, on a physical property like the density $\mathrm{n}(\mathrm{r})$ at the point $\mathrm{r}$, is limited, $|\Delta \mathrm{n}(\mathrm{r})| \leq \Delta \mathrm{n} \max (\mathrm{r}, \mathrm{R})$ provided that $\left|\mathrm{r}^{\prime}-\mathrm{r}\right|$ exceeds a given distance $R$ for all $\mathrm{r}^{\prime}$, while the chemical potential is kept fixed; and that $\Delta \mathrm{n}$ $\max (r, R)$ tends to zero with $R$ in a manner characteristic of the unperturbed system. A quantitative formulation and several illustrations will be presented.
\end{abstract}

Invited lectures

Models in heterogeneous catalysis at the atomic level

\section{H.-J. Freund}

Fritz-Haber-Institut der Max-Planck-Gesellschaft, Faradayweg 4-6, 14195 Berlin, Germany

Model catalyst systems have been prepared by growth of metal nanoaggregates on thin well-ordered oxide films of alumina, silica and magnesia. These systems lend themselves to structural and morphological characterization via scanning probe microscopies and transmission electron microscopy and bridge to a certain extent the materials gap between metal single crystal studies and the investigation of real catalyst samples.

Pd particles which have been investigated in detail are anchored to the defects of the alumina support. The nature of the support defects have been studied in atomic detail. It is possible to infer direct structure-reactivity relations when simple reactions of small molecules are studied. We have applied a variety of surface sensitive techniques, both under ultrahigh vacuum as well as under ambient conditions to relate observations from surface science to those in catalysis under realistic gas pressure conditions. Examples include methanol dehydrogenation and hydrogenation of unsaturated hydrocarbons on deposited Pd nanoparticles, as well as adsorption studies on PdCo alloy.

\section{Model Hamiltonians for Better Approximations}

F. Colonna, P. Gori-Giorgi, C. Gutle, A. Savin*, J. Toulouse

Laboratoire de Chimie Théorique CNRS and Université Paris VI 4, place Jussieu, F-75252 Paris, France

The formalism of Kohn and Sham uses a specific (model) hamiltonian which highly simplifies the many-electron problem to that of non interacting fermions. The theorem of Hohenberg and Kohn tells us that, for a given ground state density, this hamiltonian is unique. In principle, this density can be chosen as that of the real, interacting system. To obtain the energy, or other properties of the real, interacting system, approximations are needed. Working with non interacting fermions is an important simplification, but it may be easier to produce approximations with different choices of the model hamiltonian. The feature that the exact density is (ideally) reproduced can be kept in the newly defined fictitious systems.

Using model hamiltonians having the same form as the physical ones, viz., being built of one- and two-body operators allows for approaching the physical hamiltonian arbitrarily close, and thus a systematic reduction of the approximations due to the corrections to the model. In one example, the corrections to the model hamiltonian describe only short range interactions, in another, the role of high lying state. These corrections can be expressed with approximate density functionals, or explicit models of the two-body density. The price to pay is a multi-configuration calculation, but this will be less heavy than for the real hamiltonian for an adequately chosen model.

Some exact properties can be incorporated into the approximations. There remains some arbitrariness to generate approximations whose validity can only be assessed by comparing to experimental data, or heavy, but highly accurate calculations. Some applications to small atoms and molecules show that relatively simple approximations are able to improve both on usual density functional, as well as on usual multi-configuration Hartree-Fock calculations.

Invited lectures

Electrical and Optical Properties of $\pi$-Conjugated Materials: A DFT Perspective

Jean-Luc Brédas,${ }^{1,2}$ Veaceslav Coropceanu, ${ }^{1}$ Demetrio A. da Silva Filho, ${ }^{1}$ Sigifredo Carrera-Sanchez, ${ }^{1}$ David Beljonne, ${ }^{2,1}$ and Jérôme Cornil, ${ }^{2,1}$

\author{
${ }^{1}$ School of Chemistry and Biochemistry \\ Georgia Institute of Technology \\ Atlanta, Georgia 30332-0400 \\ jean-luc.bredas@chemistry.gatech.edu \\ and \\ ${ }^{2}$ Laboratory for Chemistry of Novel Materials and Center for \\ Research in Molecular Electronics and Photonics \\ Université de Mons-Hainaut \\ B-7000 Mons, Belgium
}

Conjugated organic oligomer and polymer materials are being increasingly considered for incorporation as the active semiconductor elements in devices such as light-emitting diodes, photo-voltaic cells, or field-effects transistors. In the operation of these devices, electron-transfer processes play a key role, for instance in the form of charge transport, charge separation, or charge recombination. Here, we provide a theoretical description of electron-transfer phenomena based on Marcus electron-transfer theory and full quantummechanical extensions thereof, which allows us to provide a molecular, chemically-oriented understanding [1].

In this presentation, we will focus on the parameters that impact the mobility of charge carriers, that is the electronic coupling within chains and between adjacent chains and the reorganization energy of the chains upon ionization. Materials under study include conjugated oligomers such as oligoacenes and oligoarylenes. In all instances, we will illustrate how DFT methods can be exploited to provide critical information on the efficiency of the electron-transfer processes.

[1] J.L. Brédas, D. Beljonne, V. Coropceanu, and J. Cornil, "Charge-Transfer and Energy-Transfer Processes in $\pi$-Conjugated Oligomers and Polymers", Chemical Reviews, 2004, 104, 4971-5004. 
Invited lectures

The kinetic energy functional in DFT: Exact results for model systems and plausible approximations for actual ones

Eduardo V. Ludeña ${ }^{1}$, Daniel Gómez ${ }^{1}$, Valentin V. Karasiev ${ }^{1}$, Jesus M. Ugalde $^{2}$, Xabier Lopez ${ }^{2}$, Lorenzo Echevarria ${ }^{1}$, and Pedro Nieto ${ }^{1}$

Centro de Química, Instituto Venezolano de Investigaciones Científicas, IVIC, Apartado 21827, Caracas 1020-A, Venezuela ${ }^{1}$

Kimika Fakultatea, Euskal Herriko Unibertsitatea, Posta Kutxa 1072, 20080 Donostia, Euskadi, Spain ${ }^{2}$

We re-examine the question ${ }^{1}$ of the locality of the functional derivative (Fréchet vs. Gâteaux) of the non-interacting kinetic energy functional $T_{s}[\rho]$ and show that when $T_{s}[\rho]$ is constructed from a set of densitydependent orthonormal orbitals, such as those generated by local-scaling transformations, the controversy is solved without resorting to a modified form of the functional. ${ }^{2}$

We present exact electronic kinetic energy functionals for the Hookean atom $^{3}$ both with infinite and finite (non-Born-Oppenheimer case) nuclear mass as well as for the electronic and nuclear kinetic energy functionals for recently obtained non-Born-Oppenheimer Hookean models of the $\mathrm{H}_{2}$ molecule. ${ }^{4}$

We discuss the use of local-scaling transformations for the generation of plausible approximate non-interacting electronic kinetic energy functionals for atoms. We show that these functionals lead to shell structure. We discuss the construction of kinetic energy functionals for molecules using as building blocks those of atoms. ${ }^{5,6}$

[1] R.K. Nesbet, Phys. Rev. A, 2001, 65, 010502.

[2] I. Lindgren, S. Salomomson Phys. Rev. A, 2003, 67, 056501.

[3] E.V. Ludeña et al., Int. J. Quantum Chem., 2004, 99, 297.

[4] E.V. Ludeña et al., J. Chem. Phys, 2005, (in press).

[5] V.V. Karasiev et al., Phys. Rev. A, 2000, 65, 062510.

[6] E.V. Ludeña et al., Theor. Chem. Acc., 2003, 110, 395

\section{Invited lectures}

DFT and time-dependent DFT methods with state- and orbital-dependent functionals

\section{A. Görling, P. Carrier, F. Della Sala, W. Hieringer, C.} Jamorski-Joedicke, S. Rohra, and M. Weimer

Universität Erlangen-Nürnberg, Lehrstuhl für Theoretische Chemie, Egerlandstr.3, 91058 Erlangen, Germany

DFT and time-dependent DFT methods with state- and orbital-dependent functionals for molecules and periodic systems are discussed [1]. Selfconsistent effective exact-exchange Kohn-Sham (KS) calculations for excited states within the open-shell localized Hartree-Fock framework [2] based on the generalized adiabatic connection KS approach are presented. Attempts to treat static correlation via multi-reference DFT procedures are discussed. The performance of a correlation functional based on the interaction strengths integration [3] is investigated for Van-der-Waals systems. Exact-exchange calculations for band structures and optical properties of one-dimensional periodic systems and solids $[4,5]$ are presented. The problem to treat charge transfer excitations is analyzed and possible solutions via orbital-dependent exchange-correlation kernels are discussed.

[1] A. Görling J. Chem. Phys., in press.

[2] F. Della Sala and A. Görling J. Chem. Phys., 2003, 118, 10439 .

[3] M. Seidl, J. P. Perdew, and S. Kurth Phys. Rev. Lett., 2000, 84,5070 .

[4] M. Städele, J. A. Majewski, P. Vogl, and A. Görling Phys. Rev. Lett., 1997, 79, 2089.

[5] Y.-H. Kim and A. Görling Phys. Rev. Lett., 2002, 89, 096402.
Invited lectures

\section{Post-DFT: Beyond the One-Particle Density}

\section{Peter M.W. Gill}

\section{Australian National University, Canberra ACT 0200, Australia}

Although Kohn-Sham density functional theory (DFT) is a popular and widely used tool within the computational chemistry community, it is certainly not perfect and many research groups are striving to develop better functionals. This has produced a wide array of methods and it is often hard to know which of these will be optimal for any particular problem.

There also exist a number of accurate but expensive wavefunctionbased methods, including $\operatorname{CCSD}(\mathrm{T}), \mathrm{G} 3$, W2 and others. These are usually effective at estimating the correlation energy $E_{\text {corr }}$ but their computational costs often limit their applicability to rather small molecular systems.

Recently, we have introduced a third class of methods that lies between the density-based and wavefunction-based theories and we have called these Hartree-Fock-Wigner (HFW) models. The key ingredient in these approaches is a conjecture that the Wigner intracule $W(u, v)$ of a molecular system (a function that gives the quasi-probability of finding two electrons separated by $u$ in position space and $v$ in momentum space) is related to $E_{\text {corr }}$ by

$$
E_{\text {corr }}=\int_{0}^{\infty} \int_{0}^{\infty} W(u, v) G(u, v) \mathrm{d} u \mathrm{~d} v
$$

The correlation kernel $G(u, v)$ is a universal, but as yet unknown, function.

Because they are explicitly based on two-electron information, HFW models are intrinsically more flexible than DFT models and are capable of modelling long-range correlation effects, such as dispersion energies and dissociation of odd-electron bonds, where standard DFT methods fail.

I will review the performance of HFW models for various chemical and physical properties and attempt to indicate the strengths and weaknesses of the approach

\section{Invited lectures}

Calculation of EPR Parameters for Radicals and Transition Metal Complexes. Density Functional Theory versus Simplified Correlated ab initio Methods

\section{Frank Neese}

Max-Planck Institut für Bioanorganische Chemie, Stiftstr. 34-36, D45470 Mülheim an der Ruhr, Germany

The quantum chemical modeling of EPR parameters has seen significant advances in recent years. ${ }^{[1]}$ Calculations based on density functional theory are at the stage were they give almost quantitatively accurate results for organic radicals and are a powerful complement and aid to experimental investigations. For open-shell transition metal complexes, the situation is more complicated. We have developed a number of DFT based methods to predict EPR parameters (g-tensors, hyperfine couplings, quadrupole couplings, zero-field splittings) and implemented them into the general purpose program package ORCA. ${ }^{[2]}$ Recent advances allow the simulation of the environment through continuum dielectric $^{[3]}$ and $\mathrm{QM} / \mathrm{MM}^{[4]}$ approaches, more accurate spin-orbit coupling operators $^{[5]}$ and the inclusion of scalar relativistic effects. ${ }^{[6]}$ The use of these methods in practical calculations will be illustrated. ${ }^{[4-7]}$ In addition, new developments allow us to calculate EPR parameters using simplified multireference $a b$ initio methods even for reasonably large molecules. ${ }^{[8]}$ These methods are often more accurate than DFT and are not necessarily much more expensive.

[1] (a) M. Kaupp, in EPR Spectroscopy of Free Radicals in Solids. Trends in Methods and Applications, edited by A. Lund and M. Shiotani (Kluwer, Dordrecht, 2002) (b) F. Neese and E. I. Solomon, in Magnetoscience - From Molecules to Materials. Vol IV, edited by M. Drillon and J. S. Miller (Wiley, New York, 2003), Vol. 4, pp. 345 (c) Neese, F. Curr. Op. Chem. Biol., 2003, 7, 125-135 [2] (a) Neese, F. J. Chem. Phys., 2001, 115, 11080-11096. (b) Neese, F. J. Chem. Phys., 2003,117, 39393948 (c) Neese, F.; Wolf, A.; Reiher, M.; Fleig, T.; Hess, B.A. J. Chem. Phys., in press [3] Rajendran, A.; Sinnecker, S.; Klamt, A.; Diedenhofen, M.; Neese, F. submitted [4] Schöneboom, J.; Neese, F.; Thiel, W. J. Am. Chem. Soc., 2005, ASAP article [5] Neese, F. J. Chem. Phys., 2005, 122, 034107/1-13 , A.V. Carducci, M.A.; Raitsimring, A.M.; Enemark, J.H. , A. Step, L; Bill, E.; Neese, F. Inorg. Chem, 2005, J.H. 2245

[7] Benisvy, L.; Bittl, R.; Bothe, E.; Garner, C.D.; McMaster, J.; Ross, S.; Teutloff, C.; Neese, F. Angew. Chem. Int. Ed, submitted

[8] (a) Neese, F. J. Chem. Phys., 2003, 119, 9428-9443 (b) Neese, F. Mag. Res. Chem., 2004, 42, S187. S197 
Invited lectures

Molecular Photoelectrochemical Processes: a DFT Point of View

Carlo Adamo

Laboratoire d'Electrochimie et Chimie Analytique, CNRS UMR 7575 Ecole Nationale Supérieure de Chimie de Paris, 11 rue $\mathrm{P}$. et M. Curie, F-75231 Paris Cedex 05

With the development of new theoretical and computational tools in the last decades, the ab-initio modelling is nowadays becoming a valuable and reliable means for the prediction and interpretation of the chemical reactivity and properties even of chemically interesting (medium to large size) systems. Amongst the different theoretical models, Density Functional Theory (DFT) has emerged as one of the most promising due to its favourable balance between accuracy and computational burden. This fact, together with the possibility of including environmental effects, such as solvent or adsorption on surfaces, or dynamics, on the same theoretical ground, allows the use of DFT in order to fully describe the reactivity of large systems. Here we present a review of our DFT applications to two classes of electrochemical processes. The first one is related to the inorganic dyads expected to undergo intramolecular photoinduced electron transfer and to form charge separated state, of pivotal interest in many applications, including solar energy conversion and molecular electronics [1-3]. The second example is related to the study of some electrocatalytic reactions [4]. Starting from these examples, some methodological aspects concerning the application and development of DFT will be enlightened, with a particular care to the use of recent, yet approximate, exchange-correlation functionals

[1] J.F. Guillemoles, V. Barone, L. Joubert, C. Adamo J. Phys. Chem. A 2002, 106, 11354.

[2] I. Ciofini, P.P. Lainé, F. Bedioui and C. Adamo, J. Am. Chem. Soc. 2004, 12610763

[3] P.P. Lainé, I. Ciofini, P. Ochsenbein, E. Amouyal, C. Adamo and F. Bedioui Chem. Eur. J., in press

[4] I. Ciofini, F. Bedioui, J.H. Zagal, C. Adamo Chem. Phys. Lett. 2003, 376. 690 .

Invited lectures

New Developments in Linear Scaling Density Functional Theory:

The perturbed Projector for Ab-Initio Response Theory and Hybrid HF/DFT for the Condensed Phase

\section{Matt Challacombe}

(Mchalla@LANL.Gov)

Theoretical Division, Group T-12

Los Alamos National Lab, New Mexico, 87544

In this talk I will present several developments of use in computational Density Functional Theory. First, I will describe an $\mathrm{O}(\mathrm{N})$ method for the computation of static response properties, and demonstrate linear scaling through fourth order for response to a static electric field. Also, I will introduce a linear scaling, translationally invariant implementation of the gamma-point Hartree-Fock exchange interaction, and describe its use in large scale condensed phase calculations at the hybrid HF/DFT level, employing functionals such as PBE0 and B3LYP.
Invited lectures

110

\section{PROGRESS AND PROBLEMS IN ab initio dft FOR GROUND AND EXCITED STATES}

Rodney J. Bartlett*, Igor Schweigert, Victor Lotrich, Denis Bokhan

Quantum Theory Project, University of Florida, Gainesville, FL, 32611-8435

$\mathrm{Ab}$ initio dft has the following properties: it converges to the right answer in the correlation and basis set limit, like ab initio wavefunction methods converge to the full CI.. It uses as functionals, orbital dependent expressions for the exchange and correlation energies that we know are right, at least to some order of perturbation theory; The equations are solved in a basis set usually of gaussian functions as do most wavefunction methods, so there is no numerical quadrature; yet its exchange-correlation operators are local, multiplicative. The cornerstone is the optimized effective potential approach which has a long history for the exchange, but we also use it to define correlation potentials. The correlation potentials have the right behavior unlike any currently applied hybrid (HDFT), gradient corrected (meta-GGA, GGA), or local density (LDA) method. Many results will be shown for potentials, potential energy curves, correlation energies, ionization potentials, etc. In particular, $a b$ initio $d f t$ accurately describes van Der Waals' interactions highly accurately. This attests that dft when done correctly embraces these weak interactions.

As our approach is highly flexible, the $\mathrm{V}_{\mathrm{XC}, \mathrm{S}}$ can also be made to include nonlocal operators in a straightforward way, introducing a new rational for 'hybrids' that would use some percentage of non-local HF exchange. Once realistic potentials are introduced, kS-DFT's eigenvalues do offer an approximation to the systems ionization potentials, yet the zeroth order approximation to an excitation energy, $\left\|_{I}-\right\|_{\text {a }}$, remains an excellent approximation to the TDDFT solutions when a local exchange operator is used. To the contrary, $\|_{\mathrm{a}}$ is an approximation to an electron affinity when the HF non-local potential is used in ab initio $d f t$, making this energy difference appropriate to a charge transfer state. Further ramifications and results for excited states will be presented, including the first presentation of the kernel of the OEP2 correlation potential, and its numerical results.

Invited lectures

112

Large-Scale Condensed Matter Calculations with the Gaussian and Augmented-Plane-Wave Method

Jürg Hutter, Marcella Iannuzzi, Thomas Chassaing

Physical Chemistry Institute, University of Zurich,

Winterthurerstrasse 190, CH-8057 Zurich, Switzerland

The Gaussian and Augmented-Plane-Wave (GAPW) method [1] allows for a linear scaling build-up of the Kohn-Sham matrix in all-electron calculations of condensed systems with Gaussian type basis functions. This is achieved by a partitioning of the electron density into hard and soft contributions using projector functions. The hard part of the density consists of local terms naturally expanded in a Gaussian basis, whereas the soft contributions are expanded in plane-waves by using a low energy cutoff. In combination with an efficient wavefunction optimization scheme [2] the GAPW method can be used to treat systems with several hundred atoms together with accurate basis sets. The performance of the method allows to extensively sample thermodynamic ensembles using molecular dynamics or Monte Carlo techniques. Besides accuracy and performance we will discuss recent extensions of the GAPW method to the calculation of excitation energies with time-dependent density functional response theory (TD-DFRT).

[1] G. Lippert, J. Hutter and M. Parrinello, Theor. Chem. Accounts, 1999, 103, 124.

[2] J. VandeVondele and J. Hutter, J. Chem. Phys., 2003, 118, 4365. 
Invited lectures

Invited lectures

Analysis of spin-spin interactions in transition metal clusters of biochemical interest

\section{David A. Case}

The Scripps Research Institute

La Jolla, CA 92037, USA

I will give an overview of our applications of modern tools of quantum chemistry (and in particular of density functional theory) to explore aspects of electronic stuctures of active site of metalloproteins. The focus will be on open-shell transition metal systems, especially with more than one metal site. This will include a discussion of spin-unrestricted theories, and a look at "broken symmetry" and other approaches to spin coupling among metal sites, including ways of estimating exchange interaction energies and the spectroscopic properties of coupled sites.

A second area will discuss practical methods of estimating the effects of the the protein and solvent environment. The subjects described above are essentially on "model compounds" -- fragments that mimic some aspects of the active sites of metalloproteins. Some important progress has been made in making more direct representations of active sites within their protein/solvent environment. This makes possible estimates of redox potentials and $\mathrm{pKa}$ values.

For each area, examples will largely be drawn from work (in collaboration with Lou Noodleman, Tim Lovell, and Rhonda Torres) on iron-sulfur clusters, with a focus on the MoFe protein of nitrogenase. [1-3]

[1] L. Noodleman, T. Lovell, W. Han, J. Li, F. Himo, Chem. Rev. 2004, 104, 459-508

[2] R.A. Torres, T. Lovell, L. Noodleman, D.A. Case, J. Am. Chem. Soc. 2003, 125, 1923-1936.

[3] T. Lovell, T. Liu, D.A. Case, L. Noodleman, J. Am. Chem. Soc. 2003, $125,8377-8833$

Invited lectures

Recent Developments in Conceptual Density Functional Theory : Theory and Applications

\section{F. De Proft and P. Geerlings}

Eenheid Algemene Chemie (ALGC), Vrije Universiteit Brussel (VUB), Faculteit Wetenschappen, Pleinlaan 2, 1050 Brussels, Belgium.

Next to the well-established computational advantages of density functional theory (DFT), it is the source for the introduction of a series of concepts, readily used by chemists, but previously only empirically defined. Examples are the electronegativity, hardness and softness and Fukui's frontier molecular orbital reactivity indices. Moreover, within this theory, theoretical justification was provided for several principles, such as Sanderson's principle of electronegativity equalisation and Pearson's Hard and Soft Acids and Bases and Maximum Hardness principles. This area of DFT was termed by R. G. Parr as "conceptual DFT" [1]

In this contribution, some recent theoretical developments and applications of conceptual DFT in the field of organic and inorganic chemistry are discussed. In a first part, we present an elaborate study of divalent Group 14 compounds, focussing both on their singlet-triplet gaps and their Lewis acid base properties [2] Next, we will discuss the computation of group electronegativities, hardnesses, softnesses, electrophilicities for a series of functional groups $-\mathrm{XY}_{3}$ with a Group 14 central atom $(\mathrm{X}=\mathrm{C}, \mathrm{Si}, \mathrm{Ge}, \mathrm{Sn}, \mathrm{Pb}, \mathrm{Uuq} ; \mathrm{Y}=\mathrm{H}, \mathrm{Me}, \mathrm{F}, \mathrm{Cl}, \mathrm{Br}$, I, At) [3]. Finally, attention will be focussed on a new methodology for the computation of the chemical hardness of molecules. This method will be shown to yield improved results as compared with other estimates, including the HOMO-LUMO gap and the difference of the computed vertical ionization energies and electron affinities [4]

[1] (a) R. G. Parr and W. Yang, Density Functional Theory of Atoms and Molecules, Oxford University Press, New York, 1989. (b) R. G. Parr and W. Yang, Ann. Rev. Phys. Chem. 46, 701 (1995). (c) H. Chermette, J. Comput. Chem. 20, 129 (1999). (d) P. Geerlings, F. De Proft and W. Langenaeker, Chem. Rev. 103, 1793 (2003).

[2] (a) J. Oláh, F. De Proft, T. Veszprémi and P. Geerlings, J. Phys. Chem. A 108 , 490 (2004). (b) J. Oláh, F. De Proft, T. Veszprémi and P. Geerlings, J. Phys. Chem. A 109, 1608 (2005).

[3] K. T. Giju, F. De Proft and P. Geerlings, J. Phys. Chem. A, in press.

[4] D. J. Tozer and F. De Proft, in preparation.
Invited lectures

\section{Rigorous Treatment of SingleMolecule Transport in Density Functional Theory}

\section{Kieron Burke}

Department of Chemistry and Chemical Biology, Rutgers University http://dft.rutgers.edu

In this talk, I will give a general overview of timedependent density functional theory, and mention some recent applications, including electron scattering from molecules[1]. But the main focus is on electronic transport through single molecules. I will review present calculations, and highlight three deficiencies: (1) lack of derivative discontinuity in LDA/GGA calculations, (2) missing XC corrections to Landauer formula[2], and (3) how to handle finite fields[3].

1. Continuum states from Timedependent Density Functional Theory, A. Wasserman, N.T. Maitra, and K. Burke, J. Chem. Phys. 122, 144103 (2005).

2. Zerobias molecular electronics: Exchangecorrelation corrections to Landauer's formula, K. Burke, M. Koentropp, and F. Evers, submitted, Spring 05, http://xxx.lanl.gov/abs/condmat/0502385.

3. Density Functional Theory of the Electrical Conductivity of Molecular Devices, K. Burke, Roberto Car, and Ralph Gebauer, Phys. Rev. Lett. 94, 146803 (2005). 